\title{
EDITORIAL
}

\section{Predicting survival in pulmonary arterial hypertension: time to move forward}

\author{
S. Provencher* and R. Souza ${ }^{\#}$
}

. n the early 1980s, the National Institutes of Health (NIH) established a prospective, multicenter registry to characterise the long-term outcomes of consecutive patients with primary pulmonary hypertension (PPH) [1], which included idiopathic, heritable and anorexigens-associated pulmonary arterial hypertension (PAH) [2]. Another important objective of this registry was to determine variables predicting survival. This registry, initiated when no therapy for PAH was available, confirmed the dramatic prognosis of $\mathrm{PPH}$ on supportive therapy alone. Multiple variables associated with poor prognosis were documented, including poor functional capacity, low carbon monoxide diffusion capacity, presence of Raynaud phenomenon and pulmonary haemodynamics. From this, a formula that estimated a patient's chance of survival was developed, using three hemodynamic variables (right atrial and pulmonary artery pressures and cardiac index) chosen somewhat arbitrarily. This equation was subsequently validated in an independent cohort of patients with PPH [3].

The demonstration of the devastating nature of $\mathrm{PAH}$ has justified resources for the development of new therapies, the prioritisation of $\mathrm{PAH}$ patients for lung transplantation [4] and the need for specialised PAH centres. Since the NIH registry was established, other variables have been increasingly recognised as potent prognostic factors in $\mathrm{PAH}$, including PAH type, functional capacity and other surrogate markers of right heart function. With the advent of new treatments, a better prognostication certainly led to refinements in treatment approaches, with the most aggressive options being proposed to patients with more severe disease [2]. Ultimately, the NIH equation has been useful to document higher survival on PAHspecific therapies compared with the expected survival on supportive therapy alone. In the absence of long-term randomised trials with mortality as an end-point, such observations were reassuring for the PAH community and were considered as supporting evidence of improved longterm survival with current therapies. However, the applicability of the NIH equation in the current treatment era has been questioned. Indeed, the apparent improvement in longterm survival may have resulted, in addition to treatment

\footnotetext{
*Centre de recherche de I'Institut Universitaire de Cardiologie et de Pneumologie de Québec, Université Laval, Québec, Canada. "Pulmonary Dept, Heart Institute, University of São Paulo Medical School, São Paulo, Brazil.

CORRESPONDENCE: S. Provencher, Centre de Recherche de l'Institut Universitaire de Cardiologie et de Pneumologie de Québec, Service de Pneumologie, 2725, chemin Sainte-Foy, Québec, G1V 4G5, Canada.E-mail: steve.provencher@criucpq.ulaval.ca
}

efficacy, from differences in the study population including patients' age and comorbidities, disease severity, as well as lead-time, length-time and survival biases.

In the current issue of the European Respiratory Journal, THENAPPAN et al. [5] described the contemporary (1991-2007) survival in a large monocentric registry of $\mathrm{PAH}$ patients. Similar to the NIH registry and previous reports, they confirmed that PAH related to connective tissue disease aetiology, age at diagnosis, functional capacity, right atrial pressure, cardiac index and pulmonary vascular resistance are related to long-term survival. Patients in the recent treatment era (after 2002) and responders to acute vasoreactivity testing also had a better survival in the PPH subgroup. The observed survival was significantly greater than the NIH-predicted survival. THENAPPAN et al. [5] reinforced the concept that the $\mathrm{NIH}$ equation was no longer an accurate predictor of survival in PPH. They finally developed new survival prediction equations for $\mathrm{PPH}$ patients using the same three haemodynamic variables determined a priori. These equations appropriately estimated survival when applied retrospectively to other PAH patient cohorts.

Dealing with prediction models is certainly not an easy task to accomplish. From the design of the study to the validation of the resulting model, several steps should be followed in order to minimise biases and increase external validity [6]. If a retrospective cohort has the drawback of dealing with potential problems in disease definition and missing data, a prospective cohort may limit the extrapolation of the results by restricting the inclusion criteria in order to have a more homogeneous population to follow. The study by THENAPPAN et al. [5] mixed both types of observation (17\% of patients may be considered as prospectively evaluated), thus, carrying on all mentioned characteristics for the resulting model. Another specific methodological issue that has to be carefully considered is the mathematical model of choice. The previous $\mathrm{NIH}$ equation presented a quadratic fitting as one of the steps for completing the survival model [1]. Such an approach could be considered unconventional, nowadays, since a quadratic equation usually does not properly fit a survival curve, even after combination with an exponential model, e.g. in the NIH equation, $100 \%$ survival was never present even if follow-up time equal to zero was considered. The present study used a Weibull model, which can be considered as one of the most used parametric survival models [7]. This approach certainly helped to improve fitting. The choice of variables is also a major step for building an appropriate model. THENAPPAN et al. [5] followed a conventional 
approach when performing univariate and multivariate Cox proportional hazards analyses for the whole cohort of $\mathrm{PAH}$ patients. Considering the number of the events (deaths) in the study population, such an approach provided valuable information regarding significant prognostic factors in $\mathrm{PAH}$ patients.

However, based on scientific plausibility and to make direct comparisons to the NIH model possible, THENAPPAN et al. [5] decided a priori to incorporate into their prediction equation only the same haemodynamic parameters as those in the NIH equation. The exclusion of other significant predictors of mortality obtained from the regression analyses to consider only haemodynamic parameters in the final equation is surprising [6]. If all the significant predictors had been considered, the model would certainly have better predictive properties. The lower accuracy of the model is especially problematic when applied to study populations with a different spectrum of the disease from that of the patients in whom the rule was derived [6]. For example, when applied to a previous cohort of $\mathrm{PPH}$ patients [8], the proposed equation almost perfectly predicted overall survival. However, when applied to the subgroup of patients with lower functional capacity (as assessed by a 6-min walk distance lower than the median value), it markedly overestimated survival. This suggests that, as in the NIH equation, three haemodynamic parameters are unlikely to capture the full impact of the disease on the patient and may be reliable predictors of prognosis only when applied to cohorts with similar characteristics. By excluding important prognostic information, the proposed prediction rule may have limited ability to predict outcomes in individual patients and should not be used on its own to separate high-risk from low-risk individuals. The inclusion of patients from the 1990s to develop prediction rules for the "current treatment era" is also questionable, given that the therapeutic approach has dramatically changed over the last 10 yrs and the authors documented higher survival for patients diagnosed after 2002. Finally, the authors suggested a second equation for patients that acutely responded at the vasodilator test and consequently received calcium channel blockers as first-line treatment. Considering the limited number of patients in this subgroup, any multivariate model has a high chance of over fitting, thus, critically limiting the extrapolation of the resulting equation.

The attempt of building a prediction equation, particularly in rare diseases, involves numerous difficulties [6]. The study of
THENAPPAN et al. [5] certainly represents a step forward in the clinical characterisation [9] and prognostication of $\mathrm{PAH}$ patients in the current treatment era. The study reinforces the role of different prognostic factors (such as age, connective tissue disease aetiology and functional capacity), since previous information was derived from smaller cohorts of $\mathrm{PAH}$ patients. Furthermore, the proposed equation may be of value to predict contemporary survival of similar cohorts of $\mathrm{PAH}$ patients. However, as mentioned by THENAPPAN et al. [5] this prediction rule requires prospective validation in an independent multicenter cohort and thus, should not be considered ready for clinical application before additional evidence of external validity is available.

\section{STATEMENT OF INTEREST}

A statement of interest for S. Provencher can be found at www.erj. ersjournals.com $/ \mathrm{misc} /$ statements.dtl

\section{REFERENCES}

1 D'Alonzo GE, Barst RJ, Ayres SM, et al. Survival in patients with primary pulmonary hypertension. Results from a national prospective registry. Ann Intern Med 1991; 115: 343-349.

2 Galie N, Hoeper MM, Humbert M, et al. Guidelines for the diagnosis and treatment of pulmonary hypertension. Eur Respir J 2009; 34 1219-1263.

3 Sandoval J, Bauerle O, Palomar A, et al. Survival in primary pulmonary hypertension. Validation of a prognostic equation. Circulation 1994; 89: 1733-1744.

4 Chen H, Shiboski SC, Golden JA, et al. Impact of the lung allocation score on lung transplantation for pulmonary arterial hypertension. Am J Respir Crit Care Med 2009; 180: 468-474.

5 Thenappan T, Shah SJ, Rich S, et al. Survival in pulmonary arterial hypertension: a reappraisal of the NIH risk stratification equation. Eur Respir J 2010; 35: 1079-1087.

6 McGinn TG, Guyatt GH, Wyer PC, et al. Users' guides to the medical literature XXII: how to use articles about clinical decision rules. Evidence-Based Medicine Working Group. JAMA 2000; 284: 79-84.

7 Kleinbaum DG, Klein M, eds. Survival Analysis. 2nd Edn 2010. New York, Springer Science, 2010.

8 Provencher S, Sitbon O, Humbert M, et al. Long-term outcome with first-line bosentan therapy in idiopathic pulmonary arterial hypertension. Eur Heart J 2006; 27: 589-595.

9 Souza R, Jardim C. Trends in pulmonary arterial hypertension. Eur Respir Rev 2009; 18: 7-12. 